\title{
Biomarkers Of Inflammation And Oxidative Stress In Patients With COPD And Healthy Smokers
}

\author{
P. Montuschi ${ }^{1}$, M. Aiello ${ }^{2}$, C. Buccellati ${ }^{3}$, G. Ciabattoni ${ }^{4}$, A. Chetta ${ }^{2}$, E. Clini ${ }^{5}$, G. Folco ${ }^{3}$, F. Fumagalli ${ }^{3}$, M. Losi ${ }^{5}$, D. Olivieri ${ }^{2}$, R. Pisi \\ 2 , R. Shohreh ${ }^{4}$, A. Sala ${ }^{3}$ \\ ${ }^{1}$ Catholic University of the Sacred Heart, Roma, Italy, ${ }^{2}$ University of Parma, Parma, Italy, ${ }^{3}$ University of Milan, Milan, Italy, ${ }^{4}$ University of \\ Chieti, Chieti, Italy, ${ }^{5}$ University of Modena, Modena, Italy
}

Corresponding author's email: pmontuschi@rm.unicatt.it

We measured urinary 8-isoprostane (IsoP), a biomarker of oxidative stress, and fractional exhaled nitric oxide (FeNO), a biomarker of airway inflammation, in 8 patients with COPD who were current smokers (COPD CS) (7/1 males/females, age 60 \pm 4 yrs, mean \pm SEM, FEV 1 $65.8 \pm 4.8 \%$ pred), 9 patients with COPD who were ex-smokers (COPD ES) (7/2 males/females, age $66 \pm 3$ yrs, FEV $150.6 \pm 3.6 \%$ pred), and 10 healthy smokers (HS) (9/1 m/f, $43 \pm 4$ yrs, $\mathrm{FEV}_{1} 101.7 \pm 3.1 \%$ pred). We also measured prostaglandin (PG)E2, that can have pro- and anti-inflammatory effects, in sputum supernatants in 6 COPD CS (5/1 males/females, age 60 $\pm 5 \mathrm{yrs}, \mathrm{FEV}_{1} 62.9 \pm 4.4 \%$ pred), 12 COPD ES (10/2 males/females, age $\left.67 \pm 2 \mathrm{yrs}, \mathrm{FEV}_{1} 51.3 \pm 3.3 \% \mathrm{pred}\right)$, and $4 \mathrm{HS}$ ( $3 / 1$ males/females, age $43 \pm 6 \mathrm{yrs}, \mathrm{FEV}_{1} 98.3 \pm 4.8 \%$ pred). Compared with COPD ES (268.0 $\pm 28.8 \mathrm{pg} / \mathrm{mg}$ creatinine), urinary 8-IsoP was increased in COPD CS $(506.1 \pm 85.6 \mathrm{pg} / \mathrm{mg}$ creatinine, $p<0.05)$, but not in HS (385.9 $\pm 52.2 \mathrm{pg} / \mathrm{mg}$ creatinine, $\mathrm{p}=\mathrm{ns})$, reflecting the higher degree of oxidative stress in COPD CS. FeNO was increased in COPD ES (24.8 $\pm 6.0 \mathrm{ppb})$ compared with COPD CS $(9.6 \pm 4.2 \mathrm{ppb}, \mathrm{p}<0.04)$ and HS $(8.7 \pm 1.5 \mathrm{ppb}, \mathrm{p}<0.04)$ likely reflecting the inhibitory effect of smoking on endogenous NO production and/or increased NO breakdown due to free radicals. Compared with COPD ES (138.9 \pm 22.3 $\mathrm{pg} / \mathrm{ml})$, sputum PGE 2 was increased in COPD CS $(308.0 \pm 96.6 \mathrm{pg} / \mathrm{ml}, \mathrm{p}<0.04)$ and HS $(385.5 \pm 134.6, \mathrm{p}<0.01)$ indicating a stimulating effect of smoking itself on cyclo-oxygenase activity. Different biomarkers reflect different aspects of inflammation and/or oxidative stress in patients with COPD and healthy smokers.

This abstract is funded by: PRIN-Ministry of University and Research 